on a centrifugal analyzer, since the amount of splashover depends upon the reagent as well as the suction force. No appreciable splashover should be tolerated because if the blank, standard or reference cuvettes are contaminated, all the results will be wrong.

\section{ACKNOWLEDGEMENTS}

The author is grateful to Mr. W. A. White for technical assistance, to V. A. Howe \& Co. Ltd., for the loan of the instrument and to the Department of Health and Social Security for their permission to publish this article.
REFERENCES

[1] Anderson, N.G., (1969) Analytical Biochemistry, 28, 545.

[2] Anderson, N.G., (1969) Analytical Biochemistry, 32, 59.

[3] Anderson, N.G., (1969) Science, 166, 317.

[4] Anderson, N.G., (1970) American Journal of Clinical Pathology $52,778$.

[5] Henry, P. and Saunders, R.A., (1976) . Annals of Clinical Biochemistry, 13, 384.

[6] Young, D.S. and Gochman, N., in "Standard Methods of Clinical Chemistry" volume 7 Ed. Cooper G.R. 1972 Academic Press, p. 303.

[7] Broughton, P.M.G., Buttolph, M.A., Gowenlock, A.H., Neill, D.W. and Skentelbery, R.G., (1969) Journal of Clinical Pathology 22, 278.

\title{
A microprocessor controlled liquid chromatograph/atomic absorption sampling system
}

\author{
Thomas M. Vickrey and William Eue. \\ Department of Chemistry, Texas A \& M University, College Station, Texas 77843, USA.
}

\section{Introduction}

The need for molecular characterization of metal-containing species at the trace level has led to the interfacing of high resolution chromatographic techniques with element-specific detectors $[1,2,3]$. One such system is the interfacing of liquid chromatography (LC) to atomic absorption spectroscopy (AA). The resultant instrumentation (LCAA) is capable of specific characterization (speciation) of the metalcontaining compounds and detection of the separate species at the nanogram level [3]. The application of LCAA with a graphite furnace atomic absorption spectrometer has been reported [1,2]. The use of a graphite furnace atomic absorption spectrometer as a detector for a liquid chromatograph has technical problems associated with it. However, the ability to monitor biological transport of metalcontaining species in the environment, speciate clinically important metal compounds, or to use metal complexation as a tag for nonmetal species which are otherwise difficult to detect [4], make this technique attractive.

This work presents a versatile controller for the sampling interface, using a 6800 based microprocessor system. This interface controller is software programable to operate either in the 'pulsed' mode, or in the 'total consumption' mode. The hardware and software descriptions are presented, as well as the results of sampling precision studies.

\section{Three sampling modes}

There are three basic modes for LCAA operation: survey, pulsed and total consumption. In the survey mode the sample is chromatographed, fractions collected and the collected fractions analyzed for the metal of interest. This is the simplest form of the LCAA experiment and requires only the 'human' interface. The pulsed mode operates during the chromatographic run; the eluent stream is periodically sampled and the sample dispensed into a graphite furnace for the AA analysis cycle as shown in Figure 1. The term 'pulsed'

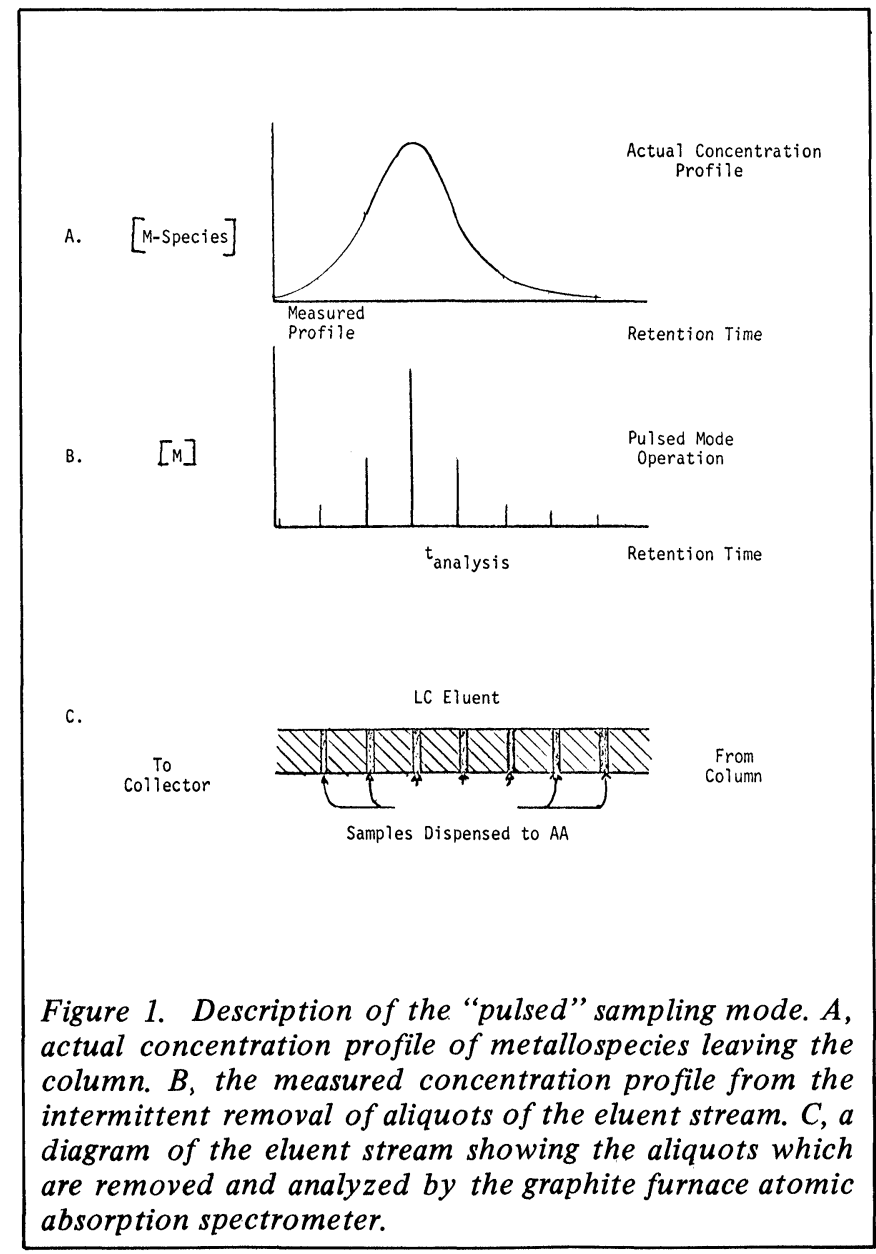

The Journal of Automatic Chemistry 
is used for this mode because the element concentration data can be obtained only once every $30-120$ seconds depending on the element of interest.

This causes the chromatogram generated by the AA detector to have the appearance of 'pulsed' concentration varying with retention time or volume. The data obtained have the form of Figure 1B. An example of a pulsed mode chromatogram is shown in Figure 2. The most direct application of the pulsed mode of LCAA operation is to determine the presence and concentration of metal-containing species with unknown retention times, or reaction mixtures where the number of products and their identity is unknown (but all products are known to contain the metal of interest). The drawbacks of pulsed mode operation are that the mobile phase flow rates that can be used are very low. This is expecially true in the case of elements which have long AA analysis times (including the cooling of the graphite furnace). The flow rate must be low enough so that the product of one

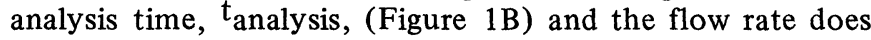
not exceed the peak width. Another related drawback is the small number of data points obtained to describe each peak's concentration profile (Figure 1B). In general, the number of data points per peak is between 4 and 8 ; also the chromatographic peak shape given by these peaks is not necessarily indicative of the shape of the concentration profile. Therefore, the third sampling method is designed to overcome these shortcomings.

The total consumption mode analyzes the total chromatographic peak by storage of the peak-containing eluent stream and subsequent dispensing of aliquots of the peak-containing eluent into the graphite furnace. In this mode the chromatographic peak is totally consumed. The temporary storage of
Step 1

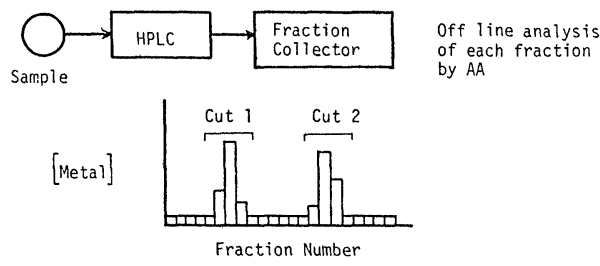

Step 2

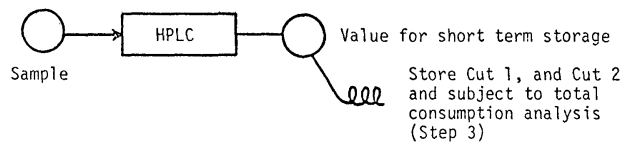

Step 3

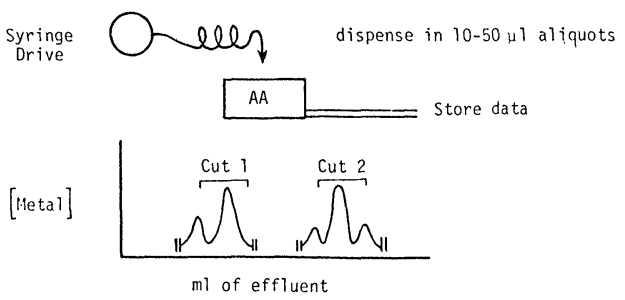

Figure 3. Scheme for initial screening of samples and subsequent total consumption analysis.

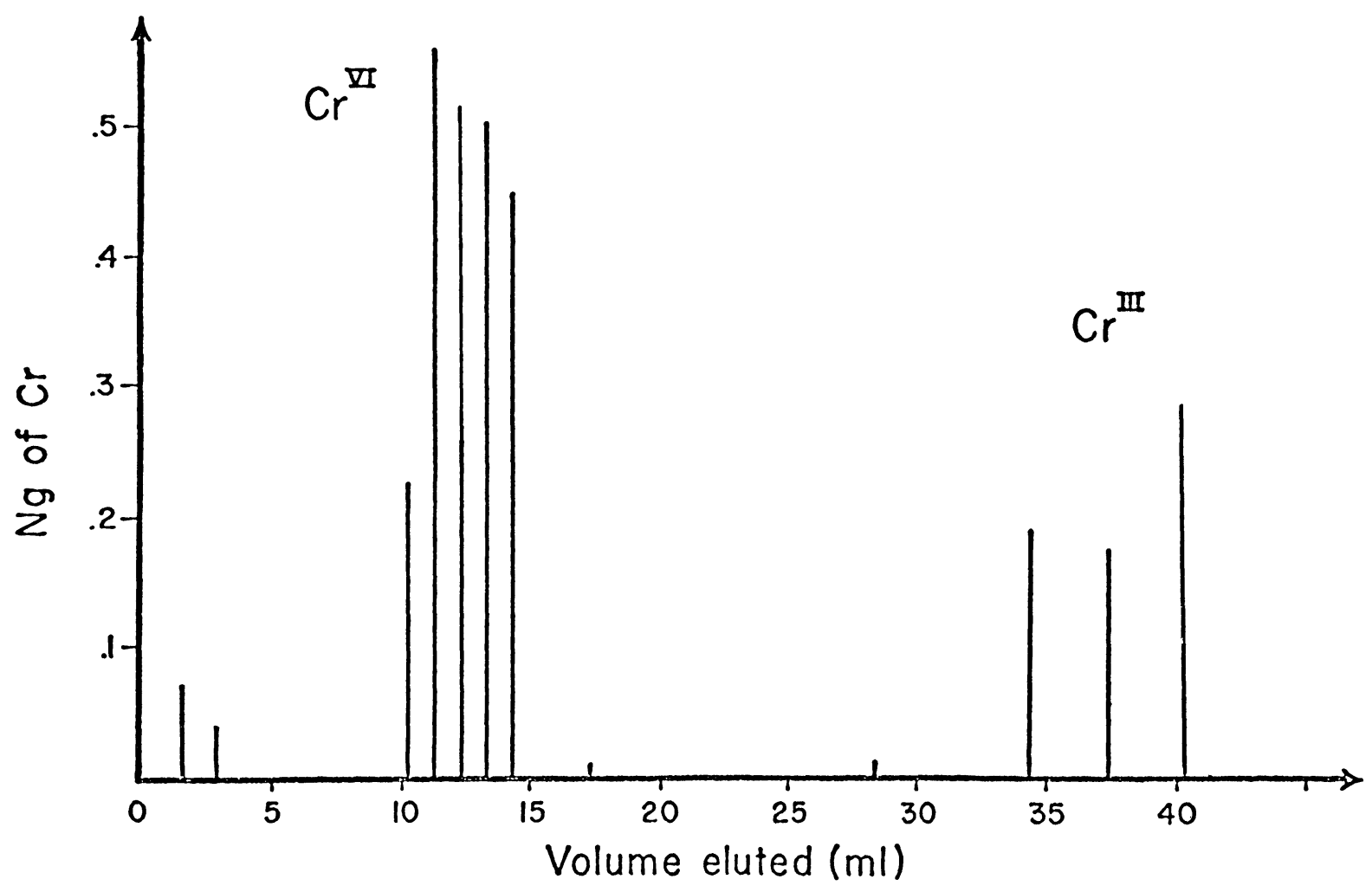

Figure 2. LCAA output for the separation of a $1: 1$ mixture of $\mathrm{Cr}(\mathrm{III})$ and $\mathrm{Cr}(V I)$ on a cation exchange column (Partisil SCX) eluting with $0.1 F$ acetate buffer, $\mathrm{pH}=4$.3. Total chromium is equivalent to $40 \mathrm{ng}$. 
the peak-containing eluent in a capillary tube allows the chromatographic information to be retained and the analysis to be performed off-line. A flow scheme for the method is shown in Figure 3. This total consumption of the chromatographic peak by the graphite furnace analyzer lowers the limit of detection, and greatly increases the number of AA data points per chomatographic peak. Since the analysis is performed off-line the restrictions on the mobile phase flow rate are removed. Data from an experiment using this sampling method are shown in Figure 4. The drawbacks to this sampling method are that the retention time of the peak musi be known (at least approximately). Also the extra

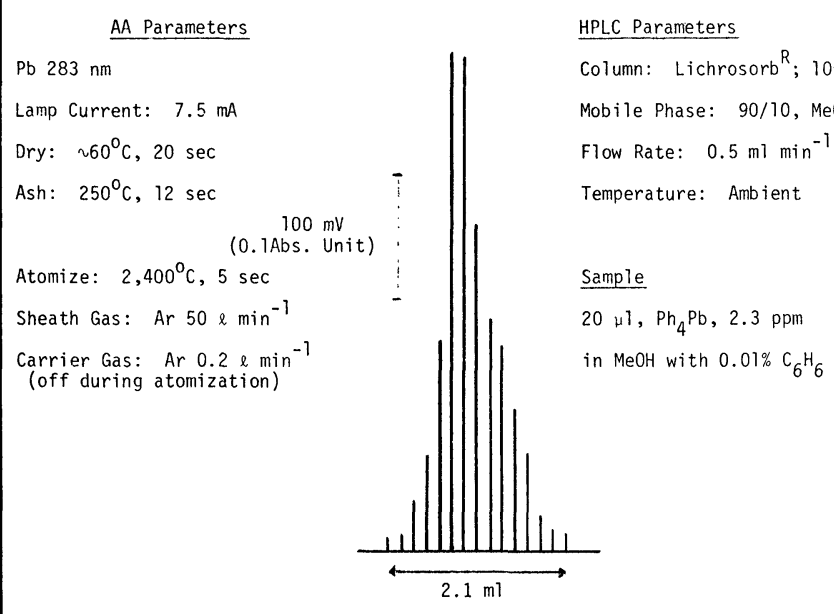

Figure 4. Total consumption mode analysis of a tetraphenyl lead sample. $L C$ and $A A$ conditions as indicated. The capillary storage tube internal volume was $2.4 \mathrm{ml}$.

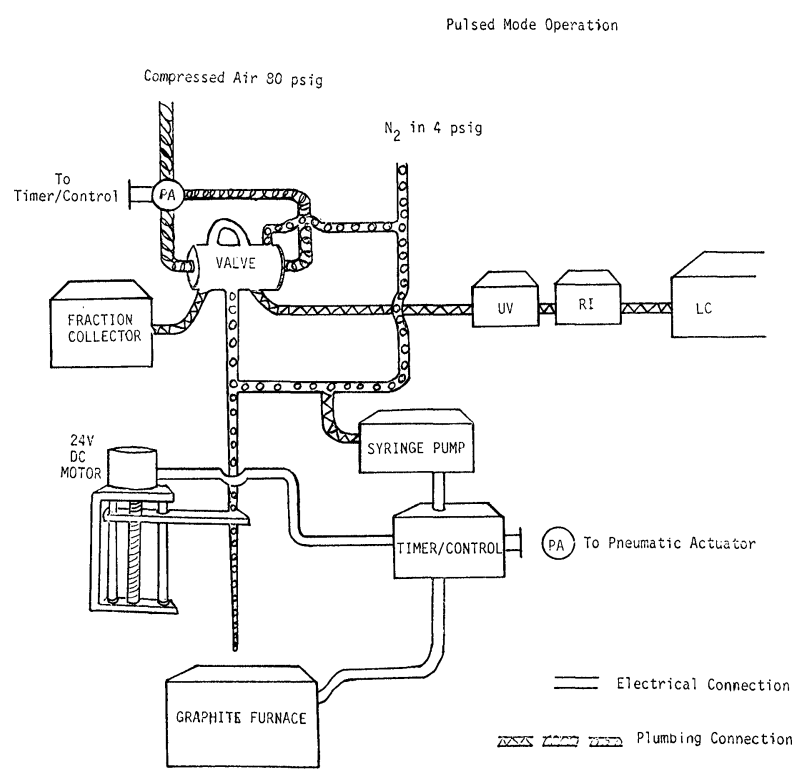

Figure 5. Schematic diagram of liquid chromatographgraphite furnace atomic absorption sampling system operating in the "pulsed mode". For total consumption mode operation, the sample storage loop is connected to the syringe pump and to the valve.

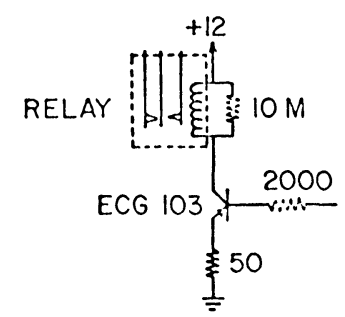

Switching Circuit

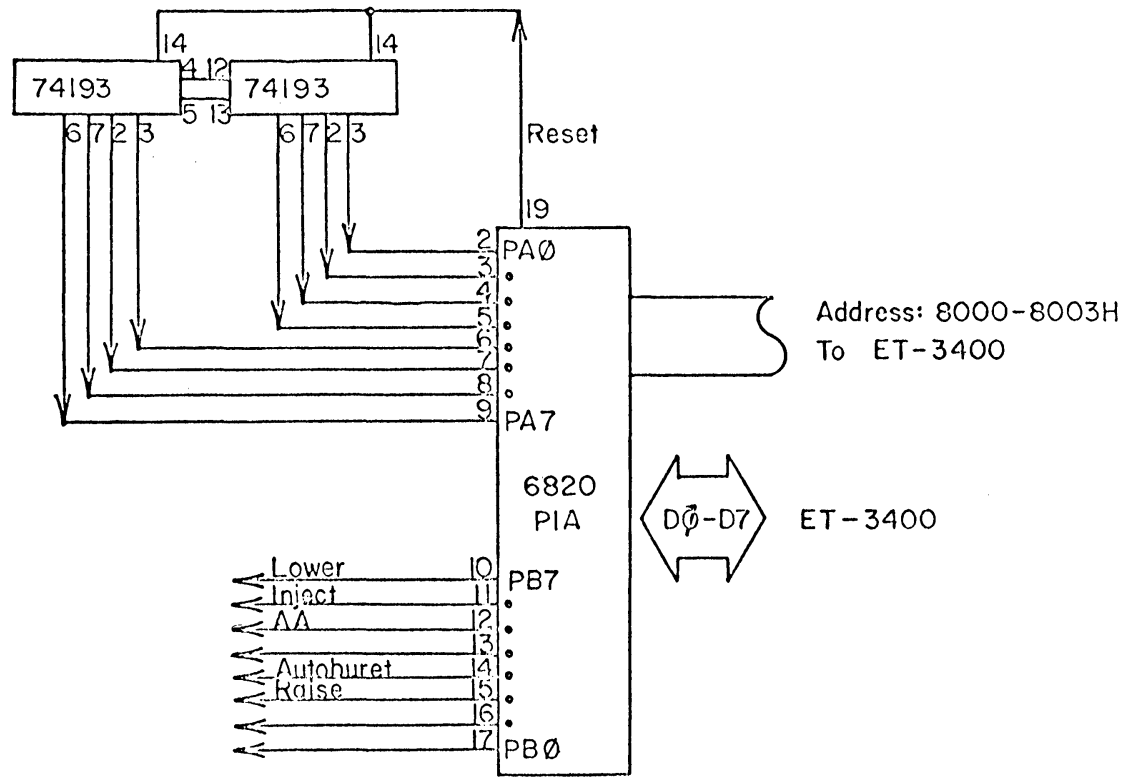

Figure 6. Control unit for the sampling system. The timer/counters are read at the top of the PIA, the control bit are put out at the bottom. For details of each function see the text. 
column broadening effects due to the large 'dead volume' of the storage tube are not negligible, but the peak width increase can be corrected in the data analysis.

The last two sampling modes described above are complementary. The pulsed mode is most useful for exploratory studies and the total consumption mode is more suited to routine analysis of samples when the identity of the components is known. The interface system used should be capable of operation in any of the modes described above.
Instrumentation and procedures

To this end, the microprocessor control LCAA interface has been developed and is shown diagramatically in Figures 5 and 6. The controller consists of (1) the Motorola 6800 based Heathkit microprocessor trainer, (2) external clock (3) electronic interface to the dispenser and to the AA, and (4) the dispenser system [1]. The microprocessor and peripheral interface adapter generate an 8 bit control pattern. This control pattern is maintained for the time programmed

Table 1. Microprocessor control program listing

\begin{tabular}{|c|c|c|c|c|c|}
\hline 00001 & \multirow{2}{*}{\multicolumn{3}{|c|}{ **INITIALIZATION OF PIA }} & AACONTR & \\
\hline 00002 & & & & & \\
\hline 00003 & 0000 & CE $0004 \quad$ START & LDX & \multirow{2}{*}{\multicolumn{2}{|c|}{$\# \$ 0004$}} \\
\hline 00004 & 0003 & FF 8000 & STX & & \\
\hline 00005 & 0006 & CE FF04 & LDX & \multirow{2}{*}{\multicolumn{2}{|c|}{ \#\$FF04 }} \\
\hline 00006 & 0009 & FF 8002 & STX & & \\
\hline 00007 & \multicolumn{5}{|c|}{$* *$ CONTROL ROUTINE LOWER RACK } \\
\hline 00008 & $000 \mathrm{C}$ & 8601 & LDA A & \#\$0001 & BIT PATTERN \\
\hline 00009 & $000 \mathrm{E}$ & B7 8002 & STA A & $\$ 8002$ & LOWER RACK \\
\hline 00010 & 0011 & C6 05 & LDA B & \#\$05 & TIME $=5$ SEC. \\
\hline 00011 & 0013 & BD 0120 & JSR INIT & & INITIALIZE CLOCK \\
\hline 00012 & \multirow{2}{*}{\multicolumn{5}{|c|}{ **CONTROL ROUTINE INJECT SAMPLE }} \\
\hline 00013 & & & & & \\
\hline 00014 & 0019 & 8602 & LDA A & \#\$02 & BIT PATTERN \\
\hline 00015 & $001 \mathrm{~B}$ & B7 8002 & STA A & $\$ 8002$ & INJECT \\
\hline 00016 & $001 E$ & C6 OA & LDA B & \#\$OA & TIME=10 SEC. \\
\hline 00017 & 0020 & BD 0100 & JSR TIMER & & TIME SEQUENCE \\
\hline 00018 & \multicolumn{5}{|c|}{ **CONTROL ROUTINE FOR FIRST DRY CYCLE } \\
\hline 00019 & 0023 & 8604 & LDA A & $\# \$ 04$ & BIT PATTERN \\
\hline 00020 & 0025 & B7 8002 & STA A & $\$ 8002$ & AA ON \\
\hline 00021 & 0028 & C6 01 & LDA B & \#\$01 & TIME=1 SEC. \\
\hline 00022 & $002 \mathrm{~A}$ & BD 0100 & JSR TIMER & & TIME SEQUENCE \\
\hline 00023 & \multicolumn{5}{|c|}{ **CONTROL SEQUENCE FOR DRYING TIME } \\
\hline 00024 & 002D & $86 \mathrm{C0}-2-10$ & LDA A & \#\$CO & BIT PATTERN \\
\hline 00025 & $002 \mathrm{~F}$ & B7 8002 & STA & $\$ 8002$ & TIME DRY \\
\hline 00026 & 0032 & C6 0A & LDA B & \#\$0A & TIME $=10$ SEC. \\
\hline 00027 & 0034 & BD 0100 & JSR TIMER & & TIME SEQUENCE \\
\hline 00028 & \multicolumn{5}{|c|}{ **CONTROL SEQUENCE FOR ENDING DRY CYCLE } \\
\hline 00029 & 0037 & 8604 & LDA A & \#\$04 & BIT PATTERN \\
\hline 00030 & 0039 & B7 8002 & LDA A & $\# \$ 04$ & BIT PATTERN \\
\hline 00031 & $003 \mathrm{C}$ & C6 01 & LDA B & \#\$01 & TIME $=1$ SEC. \\
\hline 00032 & $003 E$ & BD 0100 & JSR TIMER & & TIME SEQUENCE \\
\hline 00033 & \multicolumn{5}{|c|}{ ** CONTROL SEQUENCE FOR SECOND INJECTION } \\
\hline 00034 & 0041 & 8610 & LDA A & $\# \$ 10$ & BIT PATTERN \\
\hline 00035 & 0043 & B7 8002 & STA A & $\$ 8002$ & INJECT \\
\hline 00036 & 0046 & $\mathrm{C} 602$ & LDA B & \#\$02 & TIME $=2$ SEC \\
\hline 00037 & 0048 & BD 0100 & JSR TIMER & & TIME SEQUENCE \\
\hline 00038 & \multicolumn{5}{|c|}{ **CONTROL SEQUENCE TO RAISE RACK AND RESTART AA } \\
\hline 00039 & 004B & 8624 & LDA A & $\# \$ 24$ & BIT PATTERN \\
\hline 00040 & 004D & B7 8002 & STA A & $\$ 8002$ & RAISE \& START \\
\hline 00041 & 0050 & C6 03 & LDA B & \#\$03 & TIME $=3$ SEC. \\
\hline 00042 & 0052 & BD 0100 & JSR TIMER & & TIME SEQUENCE \\
\hline 00043 & \multicolumn{5}{|c|}{${ }^{* *}$ CONTROL SEQUENCE TO TIME AA CYCLE } \\
\hline 00044 & 0055 & 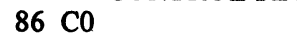 & LDA A & \#\$C0 & BIT PATTERN \\
\hline 00045 & 0057 & B7 8002 & STA A & $\pi 8002$ & TIME CYCLE \\
\hline 00046 & $005 \mathrm{~A}$ & C6 64 & LDA B & \#\$64 & TIME= 100 SEC. \\
\hline 00047 & $005 \mathrm{C}$ & BD 0100 & JSR TIMER & & TIME SEQUENCE \\
\hline 00048 & $005 \mathrm{~F}$ & 7E 0000 & JMP START & & RETURN \\
\hline 00049 & \multicolumn{5}{|c|}{ **TIME SUBROUTINE } \\
\hline 00050 & 0100 & B6 8000 TIMER & LDA A & $\$ 8000$ & GET TIME \\
\hline 00051 & 0103 & 01 & NOP & & \\
\hline 00052 & 0104 & 11 & CMP & & COMPARE B WITH A \\
\hline 00053 & 0105 & 26 F9 & BNE TIMER & & BRANCH BACK \\
\hline 00054 & 0107 & 8600 & LDA A & \#\$00 & ZERO BUFFER \\
\hline 00055 & 0109 & B7 8002 & STA A & $\$ 8002$ & OUTPUT \\
\hline 00056 & $010 \mathrm{C}$ & BD 0120 & JSR & RESET & RESET CLOCK \\
\hline 00057 & \multirow{2}{*}{\multicolumn{4}{|c|}{39 RTN }} & RETURN \\
\hline 00058 & & & & & \\
\hline 00059 & 0120 & 8608 RESET & LDA A & \#\$08 & BIT PATTERN \\
\hline 00060 & 0122 & B7 8002 & STA A & $\$ 8002$ & SET PULSE \\
\hline 00061 & 0125 & 8600 & LDA A & $\# \$ 00$ & BIT PATTERN \\
\hline 00062 & 0127 & B7 8002 & STA A & $\$ 8002$ & RESET PULSE \\
\hline 00063 & $012 \mathrm{~A}$ & 39 & RET & & RETURN \\
\hline 00064 & & & END & & \\
\hline
\end{tabular}


for each step of the analysis cycle. At the end of each step the external clock is reset as is the 8 bit control pattern for the next function. The final step is to activate the AA analysis cycle and to wait for the furnace to cool before injection of the next sample. We have found that programming a waiting time longer than the cooling time extends the life of the graphite cuvettes. The program for operation in the pulsed mode is given in Table 1 .

The second injection is a critical part of the analysis cycle for non-metals such as selenium and arsenic. The addition of a co-analyte such as $\mathrm{Ni}^{2+}$ has been shown to increase the sensitivity for these elements and reduces loss due to the volatility of these elements $[1,5]$. The syringe pump used for the second injection delivers $11.0 \pm 0.1 \mu 1 / \mathrm{sec}$ of coanalyte. The same syringe pump is used for the total consumption analysis mode. In the total consumption mode, however, the analyte is pumped out of the holding tube into the sampling valve and the dispensing of the sample then proceeds in the same fashion as in the pulsed mode of operation. The software modification for total consumption analysis involves only increasing the second injection time to 4-8 seconds and performing the second injection prior to the sample injection.

The graphite cuvette volume in the currently employed AA system is approximately $50 \mu \mathrm{l}$ and the standard pulsed mode experiment employs $37 \mu 1 \pm 1.7 \mu 1$ analyte from the sampling loop and $11.0 \mu 1+0.1 \mu 1$ of co-analyte from the syringe pump. The total consumption mode pumps $100 \mu 1$ of eluent into the sampling loop and $37 \mu 1$ are then dispensed into the graphite furnace. This 'overrun' ensures the complete filling of the sample loop and causes no problem in subsequent interpretation of data.

\section{Conclusion}

The use of an inexpensive microprocessor system adds a great deal of versatility to a previously "hard wired" LCAA sampling system [1]. Both pulsed and total consumption anaiyses are possible with only minor plumbing and software changes. The use of other microprocessor systems would require only a change in machine language. The system has the advantage of being inexpensive. The interface sampling system can be assembled for a component cost of approximately $\$ 500$ (depending on the availability of surplus equipment).

With the many advantages of performing LCAA analysis on trace level metal-containing compounds, hopefully this technique will find widespread use with investigators in the clinical, environmental, and inorganic biochemistry fields.

\section{ACKNOWLEDGEMENT}

We wish to thank April Robertson for technical assistance and the Robert A. Welch Foundation (Grant No. A-694) for financial support.

\section{REFERENCES}

[1] Vickrey, T.M. , Buren, M.S., Howell, H.E., Analytical Letters, 1978, A11, 1075-1095.

[2] Brinckman, F.E., Blair, W.R., Jewett, D.L., and Iverson, W.P. J. Chrom. Sci., 1977, 15,495-503, and references therein.

[3] Vickrey, T.M., Howell, H.E., Paradise, M.T., Analytical Chemistry, submitted.

[4] Slavin, W., Schmidt, G.J., Abstract No. 126, "Pittsburgh Conference on Analytical Chemistry and Applied Spectroscopy", Cleveland, Ohio, March 1979.

[5] Pierce, F.G., and Brown, H.R., Analytical Chemistry, 1977,49, $1417-1421$

\title{
Assessment of ENI Gemeni micro- processor-controlled centrifugal analyser
}

\author{
N. Potezny, R.G. White, and T.D. Geary
}

Institute of Medical and Veterinary Science, Box 14, Rundle Street PO, Adelaide, South Australia, 5000.

The ENI Gemeni was assessed in this institute for its suitability as a general laboratory instrument using a procedure which has been developed during the last two years for the Committee for Evaluation of Kits and Instrumentation of the Australian Association of Clinical Biochemists.

\section{Materials and Methods}

The Gemeni is a miniature centrifugal analyser consisting of an analyser module, microprocessor and work station.

The methods recommended by the manufacturer for use on the Gemeni were run in parallel with routine methods used in this laboratory. These routine methods were:
(1) Glucose
- Glucose Analyser, Yellow Springs Inst. Co. (YSI).
(2) Cholesterol - Abbott Agent Enzymatic Reagent,
(3) Calcium - Cresolphthalein complexone, SMAC, Technicon Equipment Pty. Ltd.

(4) Alkaline

(5) Phosphatase - p-nitrophenol, Beckman TR.

These methods have known accuracies and precisions. The Center of Disease Control, Atlanta, USA, (CDC) hexokinase reference method was used to check the YSI glucose analyser. The cholesterol method has been standardised against the WHO Lipid Standardisation Laboratory at CDC. The calcium method has been compared with the Cali, Young and Bowers [1] method using materials from the Massachusetts Society of Pathology with definitive values assigned by an isotope dilution technique.

The method of assessment was based upon the work of Tonks [2], Barnett and Youden [3], Logan [4], Broughton et al [5] and modified in the light of our own experience.

\section{Precision}

(a) Intrabatch imprecision was checked by the analyses of replicates in the same batch and duplicates on the patient comparisons. 


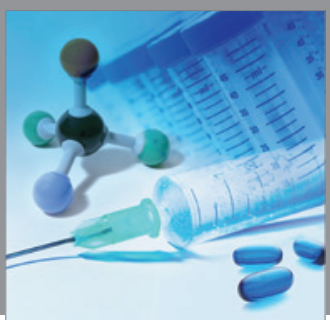

International Journal of

Medicinal Chemistry

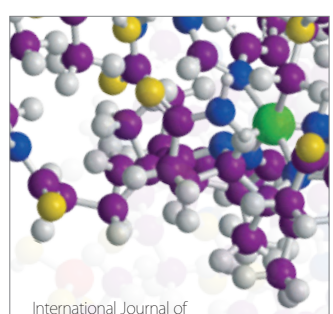

Carbohydrate Chemistry

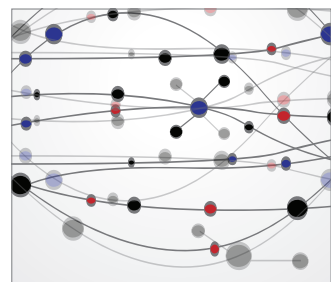

The Scientific World Journal
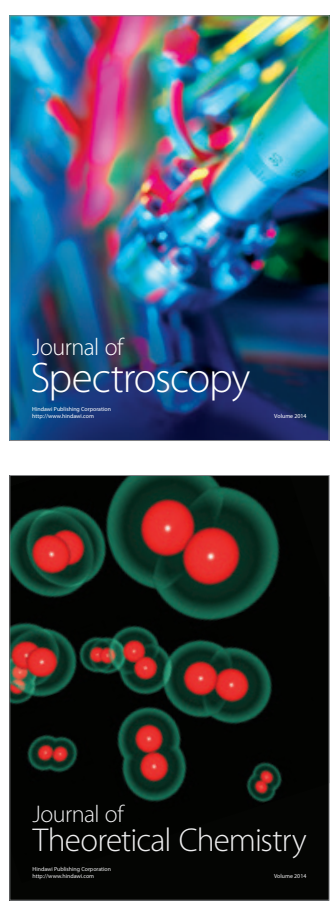
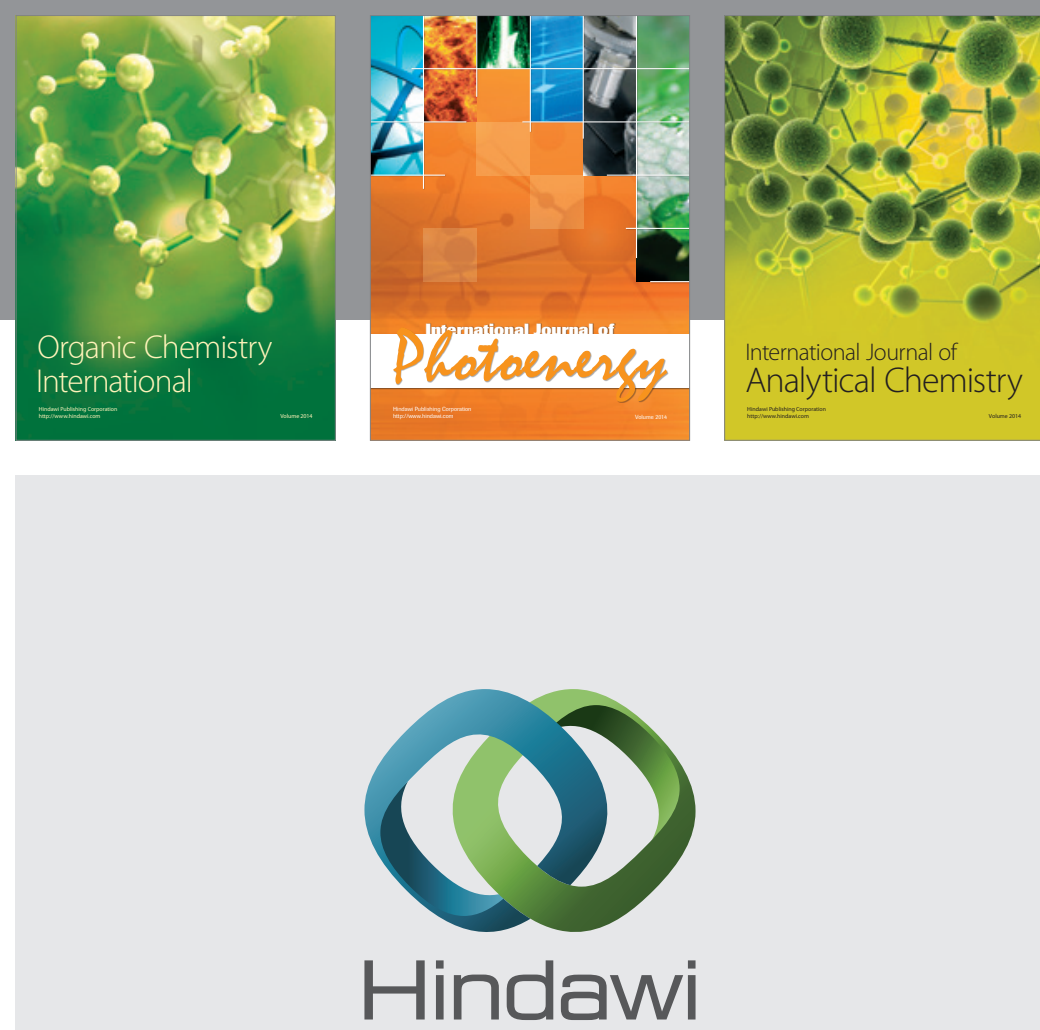

Submit your manuscripts at

http://www.hindawi.com
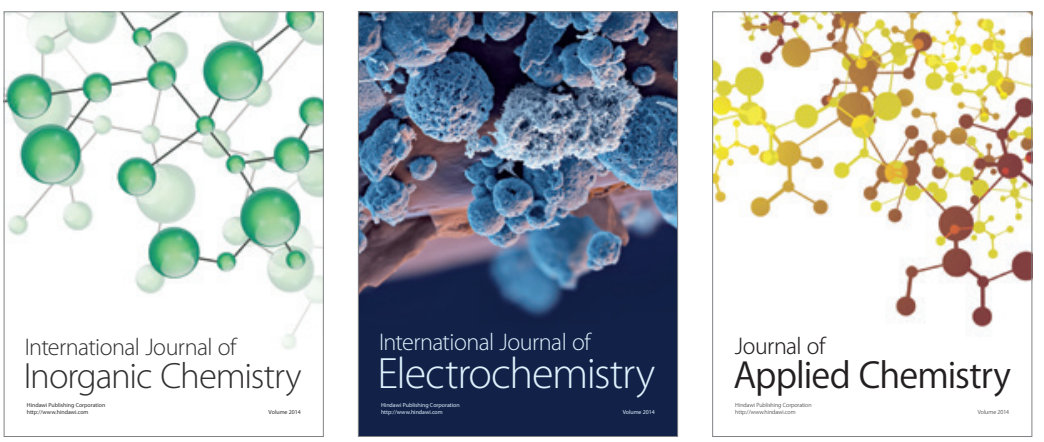

Journal of

Applied Chemistry
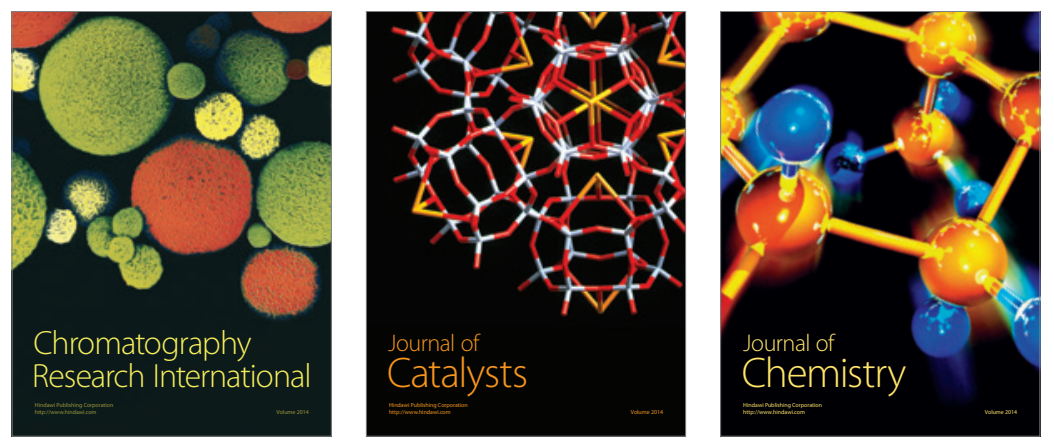
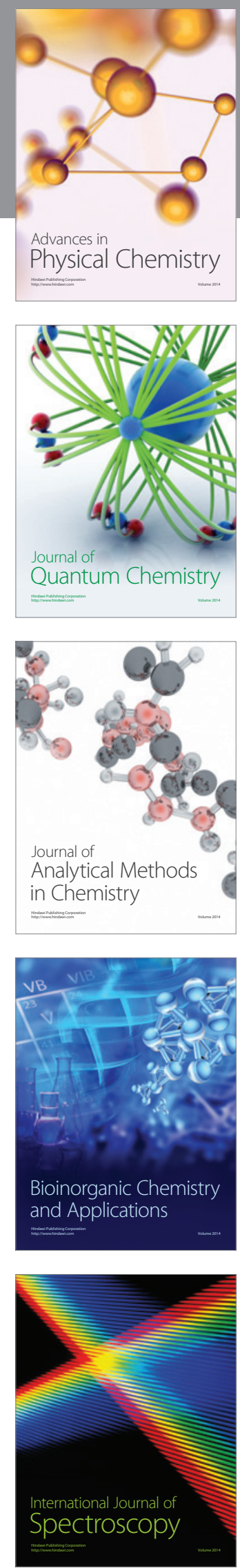